\title{
BMJ Open Effectiveness and safety of oral Chinese patent medicines as adjuvant treatment for unstable angina pectoris on the national essential drugs list of China: a protocol for a systematic review and network meta-analysis
}

\author{
Zhen Hua, ${ }^{1}$ Feng-Ting Zhai, ${ }^{2}$ Jinhui Tian, ${ }^{3}$ Cheng-Fei Gao, ${ }^{\oplus, 5}$ Peng Xu, ${ }^{6}$ \\ Fan Zhang, ${ }^{7}$ Si-jia Liu, ${ }^{8}$ Kun Dong, ${ }^{9}$ Xiao-Feng Du, ${ }^{9}$ Zhe Zhang, ${ }^{7,10}$ Guanlin Yang ${ }^{9,10}$
}

To cite: Hua Z, Zhai F-T, Tian J, et al. Effectiveness and safety of oral Chinese patent medicines as adjuvant treatment for unstable angina pectoris on the national essential drugs list of China: a protocol for a systematic review and network meta-analysis. BMJ Open 2019;9:e026136. doi:10.1136/ bmjopen-2018-026136

- Prepublication history for this paper is available online. To view these files, please visit the journal online (http://dx.doi. org/10.1136/bmjopen-2018026136).

Received 29 August 2018 Revised 18 August 2019 Accepted 20 August 2019

A Check for updates

(c) Author(s) (or their employer(s)) 2019. Re-use permitted under CC BY-NC. No commercial re-use. See rights and permissions. Published by BMJ.

For numbered affiliations see end of article.

Correspondence to Dr Zhe Zhang: pedtrainzhzh7676@163.com

Dr Guanlin Yang; yangguanlin945@gmail.com

\section{ABSTRACT}

Introduction Achieving efficacious and safe treatments for unstable angina pectoris (UAP) is still a challenging clinical problem. The availability of different oral Chinese patent medicines frequently poses a practical challenge to clinicians, namely, which one to choose as first-line regimen for treatment. This study aims to examine the comparative effectiveness and safety of oral Chinese patent medicines for UAP on the national essential drugs list of China.

Methods and analysis We will conduct a network meta-analysis (NMA) of all randomised controlled trials to evaluate the use of oral Chinese patent medicines as adjuvant for the treatment of UAP. We will explore eight electronic databases from their inception to June 2018 and search for grey literature. Primary outcomes include mortality and the cardiovascular events. Secondary outcomes include: (1) symptom improvement; (2) ECG improvement; (3) frequency of acute angina attack; (4) duration of angina; (5) adverse effects. Two independent authors will screen titles and abstracts, review full texts, extract data, assess the risk of bias using the Cochrane risk of bias tool and assess the quality of evidence and strength of the recommendations using the Grading of Recommendations Assessment, Development and Evaluation (GRADE). If adequate data are available, NMA will be performed with Bayesian analysis methods. Ethics and dissemination The NMA will help us to reduce the uncertainty of interventions and help clinicians to make optimal and more accurate therapeutic decisions for adults with UAP. Therefore, we will publish the findings of this study in a peer-reviewed journal. No ethics approval is necessary for this study based on the nature of its design.

Trial registration number CRD42018092822.

\section{INTRODUCTION}

Unstable angina pectoris (UAP) is a transitory and critical syndrome, characterised by severe transient myocardial ischaemia. ${ }^{1}$ UAP is a type
Strengths and limitations of this study

- This network meta-analysis will provide evidence of the comparative effectiveness and safety of oral Chinese patent medicines for unstable angina pectoris on the national essential drugs list of China for the first time.

- This network meta-analysis will be based on a comprehensive search strategy and selection of studies; data extraction and estimation of quality will be performed independently by two reviewers to ensure the integrity and authenticity of all relevant studies.

- We will assess the quality of evidence and the strength of recommendations for the main outcomes using the Grading of Recommendations Assessment, Development and Evaluation (GRADE) guidelines.

- This review will not cover medical databases in other languages (such as Japanese and Korean), so a language bias may exist.

of acute coronary syndrome, which is very common and often quite serious. In 2014, there were 24000 UAP principal diagnosis discharges and the corresponding number of inpatient hospital discharges was 382000 unique hospitalisations for UAP when secondary discharge diagnoses were included in the USA. ${ }^{2}$ More importantly, UAP is easily exacerbated into acute myocardial infarction, ischaemic death and severe cardiac arrhythmias which can lead to sudden death..$^{2-4}$ Therefore, we must keep in mind that treatment should begin immediately after the onset of UAP.

Recent studies have shown that the main mechanism underlying UAP initiation was plaque rupture or erosion with overlying thrombosis. This process is mainly related to 
vascular endothelial injury, the inflammatory response, platelet activation and aggregation and other relevant factors. ${ }^{5-7}$

Multiple treatment regimens and drugs are recommended in various clinical guidelines, including invasive strategies, conservative treatment, anti-ischaemic therapy, nitroglycerin, antiplatelet therapy, aspirin, beta blockers and so on. ${ }^{8-10}$ Among them, conservative treatment was performed in $76.2 \%$ of the patients with UAP. Although conservative treatment was used in patients with normal coronary angiogram, still in $77.7 \%$ of UAP with high-risk criteria it was performed. ${ }^{11}$ The aim of these treatments is to improve quality of life by reducing angina attacks and prevent cardiovascular events as well as death. However, not all of these treatments are useful for UAP. The ProAcor Study showed that 1-year mortality was still up to $7.6 \%$ and at 1-year cardiac events were noted in $14.9 \%$ even in very standard and reasonable treatments. ${ }^{12}$ And drawbacks such as drug resistance and adverse effects are issues too. ${ }^{13}$ Achieving safe and effective treatments for UAP is still a challenging clinical problem.

As early as 3000 years ago, Chinese medicine has already appeared and encompasses unique theories for aetiology, pathogenesis, diagnosis and treatment. ${ }^{14} 15$ A review has indicated that traditional chinese medicine (TCM) could be used as a complementary and alternative approach in the primary and secondary prevention of cardiovascular disease. ${ }^{16}$ In the developing and developed world, an increasing number of people accept TCM as an alternative to conventional treatments. ${ }^{18}{ }^{18}$ Oral Chinese patent medicine is an important part of TCM. And Chinese patent medicines are a kind of TCM products, which is commercialised and approved by the State Drug Supervision and Administration Department. In order to prevent and treat diseases, TCM products are processed into certain dosage forms using TCM as raw material and the theory of TCM as guide. Common dosage forms include injection, oral liquid dosage forms (solution, suspension, emulsion) and oral solid dosage forms (powder, capsule, tablet, pill). ${ }^{19}$ Oral Chinese patent medicine is the collective name of the latter two. And it has been used in clinical practice for the treatment of UAP in East and South Asia, such as China, Japan and Korea. ${ }^{20-22}$

Several systematic reviews have been conducted to examine the comparative effectiveness and safety of single oral Chinese patent medicines for UAP. ${ }^{23-25}$ As the number of oral Chinese patent medicines increases, the required number of pairwise systematic reviews would increase exponentially. Pairwise meta-analysis of multiple treatments is laborious and time-consuming. In addition, because different head-to-head oral Chinese patent medicines trials are scarce, these systematic reviews have not focused on any head-to-head comparisons of different oral Chinese patent medicines. Although these studies confirm that a single oral proprietary Chinese medicine is effective against UAP, the availability of different oral Chinese patent medicines frequently poses a practical challenge to clinicians, namely, which one to choose as first-line regimen for treatment.

The use of network meta-analysis (NMA) allows the direct and indirect comparison of different oral Chinese patent medicine therapeutic options for UAP in terms of safety and effectiveness. It is a highly valuable tool allowing indirect comparisons of multiple interventions to estimate their safety and effectiveness and rank them. ${ }^{26}$ Based on these reasons, we decided to conduct a NMA comparing oral Chinese patent medicines for UAP on the national essential drugs list of China, to examine their relative effectiveness and safety.

\section{OBJECTIVE}

This NMA aims to examine the safety and effectiveness of oral Chinese patent medicines for UAP on the national essential drugs list of China.

\section{METHODS AND ANALYSIS}

Our NMA protocol has been registered on the PROSPERO international prospective register of systematic reviews (ID=CRD42018092822). Our protocol has been prepared in accordance with the Preferred Reporting Items for Systematic Review and Meta-Analysis Protocols (PRISMA-P) guidelines. ${ }^{27}$ The final report will comply with the recommendations of the PRISMA Extension Statement for Reporting of Systematic Reviews Incorporating Network Meta-analyses of Healthcare Interventions. ${ }^{28}$

\section{Eligibility criteria}

Type of study

We will include all relevant randomised controlled trials (RCTs) regardless of blinding and concealment, as RCT is regarded as the gold criterion for examining interventions. Cross-over and cluster randomised trials will also be included. Quasi-RCTs will be excluded such as those allocating using the number of medical records. We will not include studies with the high risk of bias, such as only one researcher or the research sites are community or low level hospitals.

\section{Types of participants}

Adults (aged $\geq 18$ years) with UAP diagnosed according to the standards revised by 'Nomenclature and criteria for diagnosis of ischaemic heart disease' released by WHO in $1979^{29}$ and 'diagnosis and treatment of UAP' released by Chinese Society of Cardiovascular Diseases of Chinese Medical Association in $2000^{30}$ and $2007^{31}$ will be included. According to the risk stratification of UAP, current guidelines recommend invasive treatment regimens first for very-high-risk or high-risk patients. ${ }^{910}$ So, those very-high-risk or high-risk patients will be excluded. Patients who have undergone coronary artery bypass graft (CABG) or percutaneous transluminal coronary angioplasty (PTCA), with acute myocardial infarction or other deadly serious diseases, 
such as cancer, will be excluded, because the risk of these patients is significantly different from that of the patients with UAP. ${ }^{910}$ We will not apply restrictions with regard to gender, ethnic origin or other characteristics.

\section{Types of interventions}

Interventions involving any oral Chinese patent medicines for the treatment of UAP are eligible. These oral Chinese patent medicines could be used in combination with routine treatment regardless of dose, duration, preparation form and manufacturer. The control groups include routine treatment or another oral Chinese patent medicine plus routine treatment. The following groups are eligible: Oral Chinese patent medicine+routine treatment versus another oral Chinese patent medicine+routine treatment and Oral Chinese patent medicine+routine treatment versus routine treatment. Routine treatment includes anti-ischaemia, antiplatelet, anticoagulation, lipid-lowering and so on. As for the usage of the treatment, including the selection of the timing of intervention during UAP treatment, follow the guidelines. But the two groups of routine treatment must be the same in the same RCT. We are interested in comparing the following drugs: Shengmaiyin, Wenxinkeli, Xueshuantongjiaonang, Xuesaitongjiaonang, Yinxingye, Yindanxinnaotongruanjiaonang, Shexiangbaoxinwan, Naoxintongwan, Xinmainingjiaonang, Xueshuanxinmainingjiaonang, Shensongyangxinjiaonang, Yixinshujiaonang, Guanxinsuhewan, Diaoxinxuekangjiaonang, Tongxinluojiaonang, Dengzhanhuasupian, Naoankeli, Maixuekangjiaonang, Xuefuzhuyuwan, Fufangdanshen, Suxiaojiuxinwan, Xinkeshu, NuodiKangjiaonang and so on. ${ }^{19}$ Since there are many dosage forms of a drug, such as Shengmaiyin including Shengmaiyinjiaonang and Shengmaiyinkeli, we use Shengmai yin as a unified substitute and separate them if necessary. Figure 1 shows the network of all possible pairwise comparisons among the eligible interventions. We anticipate that any patient who meets all inclusion criteria is equally to be randomised to any of the interventions in the synthesis comparator set. Trials which allow rescue medications will be included so long as these are equally provided among the randomised arms. If Chinese patent medicines (such as suxiaojiuxinwan) were used as rescue medications in a study, and the intervention measures were not this medicine in the study, this study would be excluded. Trials that only compare different oral Chinese patent medicines without routine treatment or self-made proprietary Chinese patent medicines will be excluded.

\section{Types of outcome measures}

UAP has the highest risk of myocardial infarction and death within 2 months after the onset of disease. ${ }^{32}$ It is of great value to pay attention to the efficacy of this period. Thus, the time point we need is within 2 months of onset and those patients with the course of disease within 2 months will be enrolled.

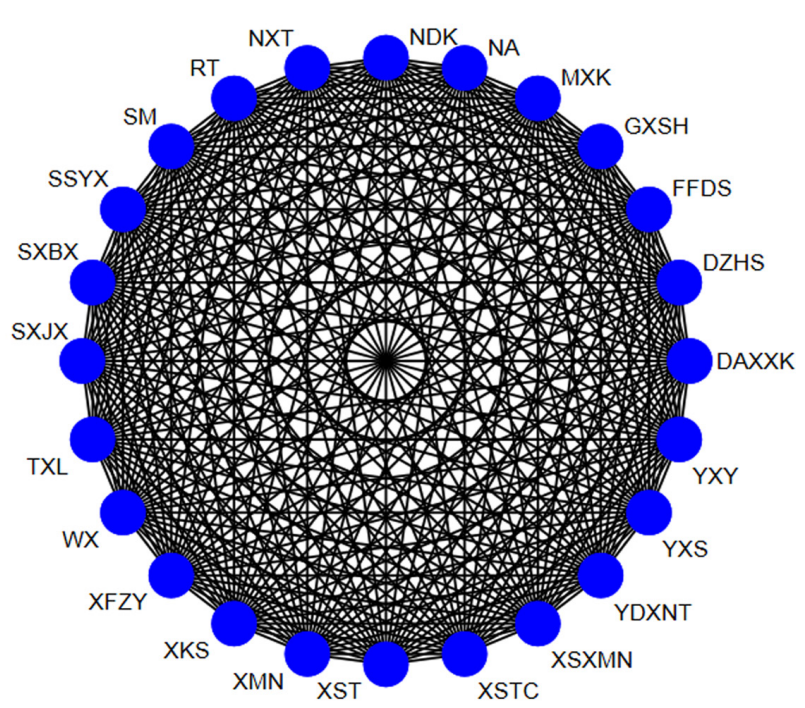

Figure 1 Network of all possible pairwise comparisons between the eligible interventions. DAXXK, Diaoxinxuekangjiaonang+RT; DZHS, Dengzhanhuasupian+RT; FFDS, Fufangdanshen+RT; GXSH, Guanxinsuhewan+RT; MXK, Maixuekangjiaonang+RT; NA, Naoankeli+RT; NDK, NuodiKangjiaonang+RT; NXT, Naoxintongwan+RT; RT, Routine treatment; SM, Shengmaiyin+RT; SSYX, Shensongyangxinjiaonang+RT; SXBX, Shexiangbaoxinwan+RT; SXJX,

Suxiaojiuxinwan+RT; TXL, Tongxinluojiaonang+RT; WX, Wenxinkeli+RT; XFZY, Xuefuzhuyuwan+RT; XKS, Xinkeshu+RT; XMN, Xinmainingjiaonang+RT; XST, Xuesaitongjiaonang+RT; XSTC, Xueshuantongjiaonang+RT; XSXMN, Xueshuanxinmainingjiaonang+RT;

YDXNT, Yindanxinnaotongruanjiaonang+RT; YXS, Yixinshujiaonang+RT; YXY, Yinxingye+RT.

\section{Primary outcomes}

The mortality

It is defined as the proportion of patients who die for any reason during the treatment (within 2 months).

\section{The cardiovascular events}

The proportion of patients with cardiovascular events (within 2 months). The cardiovascular events include endpoints fatal versus nonfatal of events (eg, miocardial infarction (MI)), different types of events (eg, Q-wave versus non-Q-wave MI), bleeding, stroke, transient ischemic attack (TIA) and other important cardiovascular outcomes (eg, CABG and PTCA).

\section{Secondary outcomes}

Symptom improvement

Measured by the total number of patients who had a remission of symptoms (including chest distress, shortness of breath and hypodynamia) between baseline and the end of treatment (within 2 months) on any standardised rating scale.

\section{ECG improvement}

Measured by the total number of patients who had a remission of ECG between baseline and the end of treatment 


\begin{tabular}{|c|c|c|}
\hline Outcomes & $\begin{array}{l}\text { Measurement of variables } \\
\text { (units) }\end{array}$ & $\begin{array}{l}\text { Statistical } \\
\text { estimates and } \\
\text { measurement of } \\
\text { association }\end{array}$ \\
\hline \multicolumn{3}{|l|}{ Primary } \\
\hline Mortality & Number of deaths & OR (95\% Crl) \\
\hline $\begin{array}{l}\text { Cardiovascular } \\
\text { events }\end{array}$ & $\begin{array}{l}\text { Number of MI, CABG or } \\
\text { PTCA }\end{array}$ & OR (95\% Crl) \\
\hline \multicolumn{3}{|l|}{ Secondary } \\
\hline $\begin{array}{l}\text { Symptom } \\
\text { improvement }\end{array}$ & Number of improvement & OR (95\% Crl) \\
\hline ECG improvement & Number of ECG improvement & OR (95\% Crl) \\
\hline $\begin{array}{l}\text { Frequency of acute } \\
\text { angina attacks }\end{array}$ & $\begin{array}{l}\text { Number of acute angina } \\
\text { attacks }\end{array}$ & MD (95\% Crl) \\
\hline Duration of angina & Time of angina & MD (95\% Crl) \\
\hline Adverse effects & $\begin{array}{l}\text { Side effects: toxic response, } \\
\text { discontinuation, digestive } \\
\text { symptoms, headache and } \\
\text { dizziness so on. }\end{array}$ & $\begin{array}{l}\text { OR or MD }(95 \% \\
\text { Crl) }\end{array}$ \\
\hline
\end{tabular}

95\% Crl, 95\% credibility interval; MD, mean difference; OR, odds ratio.

(within 2 months). Remission is defined as that the ECG returned to 'approximately normal', reached 'normal ECG' or the ST segment reduced by more than $0.05 \mathrm{mV}^{33}$

\section{Frequency of acute angina attacks}

It is defined as number of angina attacks within a specified period of time (a day or a week).

\section{Duration of angina}

It is defined as average time of angina attack within prescribed time.

\section{Adverse effects}

Measured by the total number of patients who suffered from those adverse effects between baseline and the end of treatment (within 2 months). The adverse effects include bleeding events, toxic response, discontinuation, digestive symptoms, headache, dizziness and so on.

The study with incorrect, incomplete or unavailable data of outcomes will be excluded. The definitions of the outcomes are described in table 1 . Those outcome measures including mortality, the cardiovascular events, symptom improvement, ECG improvement, frequency of acute angina attacks and duration of angina will be used to assess for effectiveness and safety assessment will be measured by incidence and severity of adverse effects. See figure 2 for full details about the data selecting and extraction of outcome (decision tree).

\section{Search methods for the identification of studies}

Search strategies have been developed through the liaison with Xiao-feng Du who is an experienced librarian at Liaoning University of Traditional Chinese Medicine Library. We will explore the following eight electronic databases: PubMed, CNKI, Cochrane Central Register of Controlled Trials (CENTRAL) in the Cochrane Library,

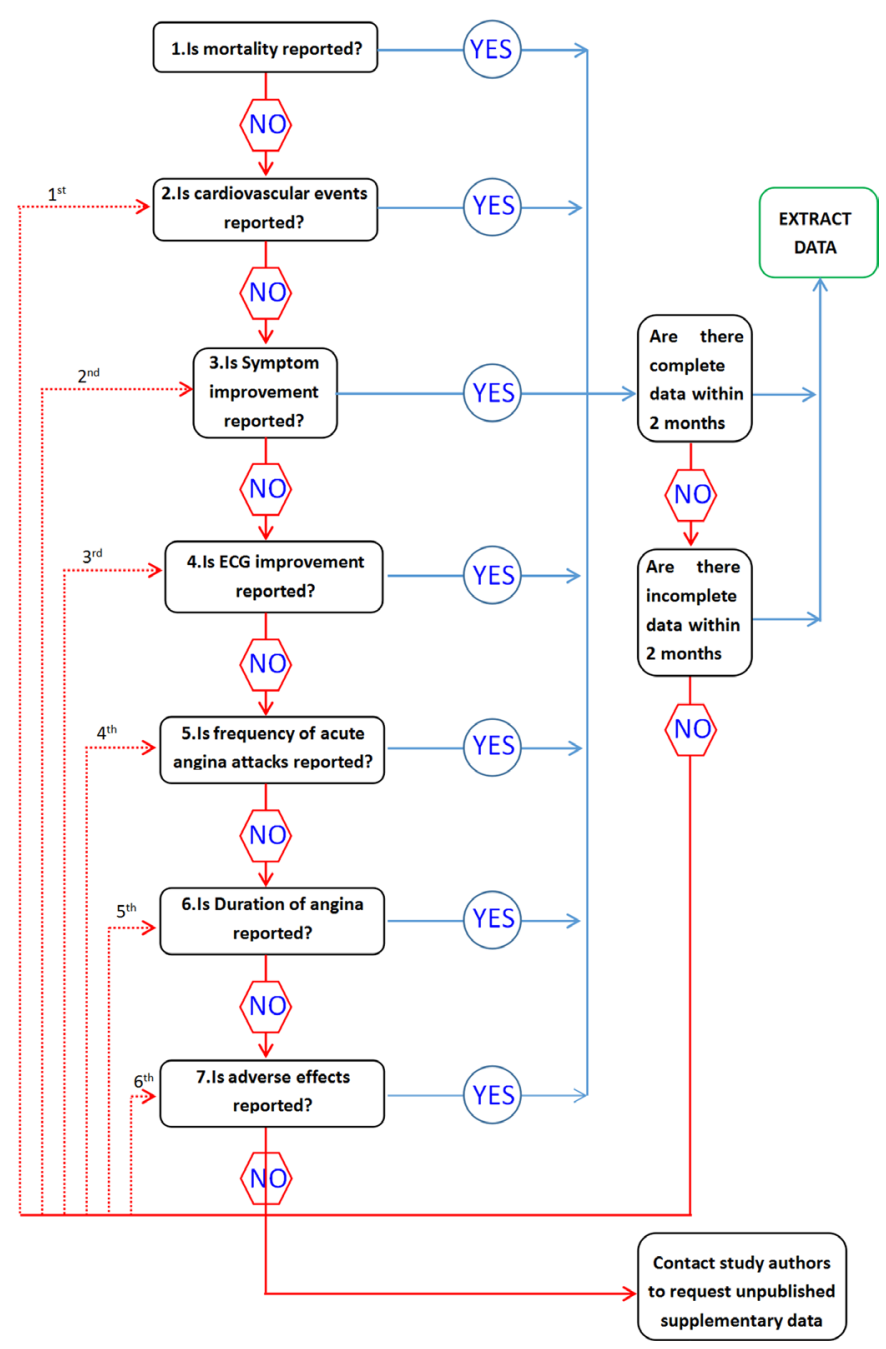

Figure 2 Decision-tree for data selecting and extraction of outcome.

EMBASE, Web of Science, Wanfang Database, VIP, SinoMed, from their inception to June 2018. The search will be conducted in English and Chinese. We will use a combination of Medical Subject Heading and free-text terms including various synonyms for UAP, Traditional Chinese Medicine and oral Chinese patent medicines on the national essential drugs list of China (table 2 details the search strategy for PubMed). We will also search ongoing trial registers in the trial registry websites (such as the Chinese Clinical Trial Registry and the US National Institutes of Health resource) to obtain grey literature. At the same time, we will also plan to perform a manual search for the reference lists of reviews captured by the initial searches. There will be no restrictions on date limit, country, publication status or year of publication.

\section{Data collection}

\section{Selection of studies}

NoteExpress literature management software (v3.2.0.7103) will be used to manage all studies. ${ }^{34}$ First, the software will be used to classify and organise the preliminary literature and exclude the repeated collection of literature. Second, two independent review 


\begin{tabular}{|c|c|}
\hline No. & Search item \\
\hline \#1 & Angina, Unstable[MeSH Terms] \\
\hline \#3 & \#1 OR \#2 \\
\hline \#4 & Medicine, Chinese Traditional[MeSH Terms] \\
\hline \#5 & $\begin{array}{l}\text { Medicine, Chinese Traditional[Title/Abstract] OR Traditional Chinese Medicine[Title/Abstract] OR Chung I Hsueh[Title/ } \\
\text { Abstract] OR Hsueh, Chung I[Title/Abstract] OR Traditional Medicine,Chinese[Title/Abstract] OR Zhong Yi Xue[Title/ } \\
\text { Abstract] OR Chinese TraditionalMedicine[Title/Abstract] OR Chinese Medicine, Traditional[Title/Abstract] OR Drugs, } \\
\text { Chinese Herbal[Title/Abstract] OR Chinese Drugs, Plant[Title/Abstract]OR Chinese Herbal Drugs[Title/Abstract] OR } \\
\text { Herbal Drugs,Chinese[Title/Abstract] OR Plant Extracts, Chinese[Title/Abstract] OR ChinesePlant Extracts[Title/ } \\
\text { Abstract] OR Extracts, Chinese Plant[Title/Abstract] }\end{array}$ \\
\hline \#6 & \#4 OR \#5 \\
\hline \#7 & $\begin{array}{l}\text { shengmai[Title/Abstract]) OR wenxin[Title/Abstract] OR xueshuantong[Title/Abstract] OR xuesaitong[Title/ } \\
\text { Abstract] OR yinxingye[Title/Abstract] OR yindan xinnaotong[Title/Abstract] OR shexiangBaoxin[Title/ } \\
\text { Abstract] OR naoxintong[Title/Abstract] OR xinmaining[Title/Abstract] OR xueshuan xinmaining[Title/Abstract] } \\
\text { OR shensongyangxin[Title/Abstract] OR yixinshu[Title/Abstract] OR guanxinsuhe[Title/Abstract] OR diao } \\
\text { xinxuekang[Title/Abstract] OR xinxuekang[Title/Abstract] OR tongxinluo[Title/Abstract] OR dengzhanhuasu[Title/ } \\
\text { Abstract] OR naoan[Title/Abstract] OR maixuekang[Title/Abstract] OR xuefu zhuyu[Title/Abstract] OR } \\
\text { fufangdanshen[Title/Abstract] OR compound danshen[Title/Abstract] OR suxiaojiuxin[Title/Abstract] OR } \\
\text { xinkeshu[Title/Abstract] }\end{array}$ \\
\hline \#8 & \#6 OR \#7 \\
\hline
\end{tabular}

authors (Peng Xu and Kun Dong) will screen the titles and abstracts of each study retrieved to identify relevant studies based on the eligibility criteria. Then, we will obtain the full text of all the studies potentially suitable for the NMA and review them for further assessment. In this process, the two people will screen the titles, abstracts and the full text of each study, respectively, make decisions independently and then compare their selection of studies. Once the conclusions of the two evaluators are inconsistent, the differences are resolved through discussion. If the agreement cannot be reached, we will seek the help of a third author (Guan-lin Yang or Zhe Zhang) for judgement and arbitration. And if the disagreement is caused by insufficient information of the literature, it is necessary to classify the literature into the category of waiting for evaluation and then decide whether the literature should be included after sufficient additional information is added. In an excluded studies list, we will report the reasons for exclusion of studies in the full-text review.
A flowchart that depicts the search process, including a reference list of all excluded studies, will be included. The proposed structure for the flowchart is shown in figure 3.

\section{Data extraction and management}

Data from the studies will be extracted by two review authors (Peng Xu and Kun Dong) independently using a pretested Microsoft Excel data extraction form. The data items we plan to extract include:

1. Study characteristics (including authors, journal, publication year, method of randomisation and blinding method).

2. Participants (sample size, age, duration of disease, disease diagnostic criteria, type of UAP and symptoms and signs).

3. Intervention (type of therapy, dosage forms, clinical doses and course of treatment).

4. Control (type of therapy, the course of treatment, dosage forms and clinical doses).

5. Outcomes (results, types of outcome measures and adverse events). 


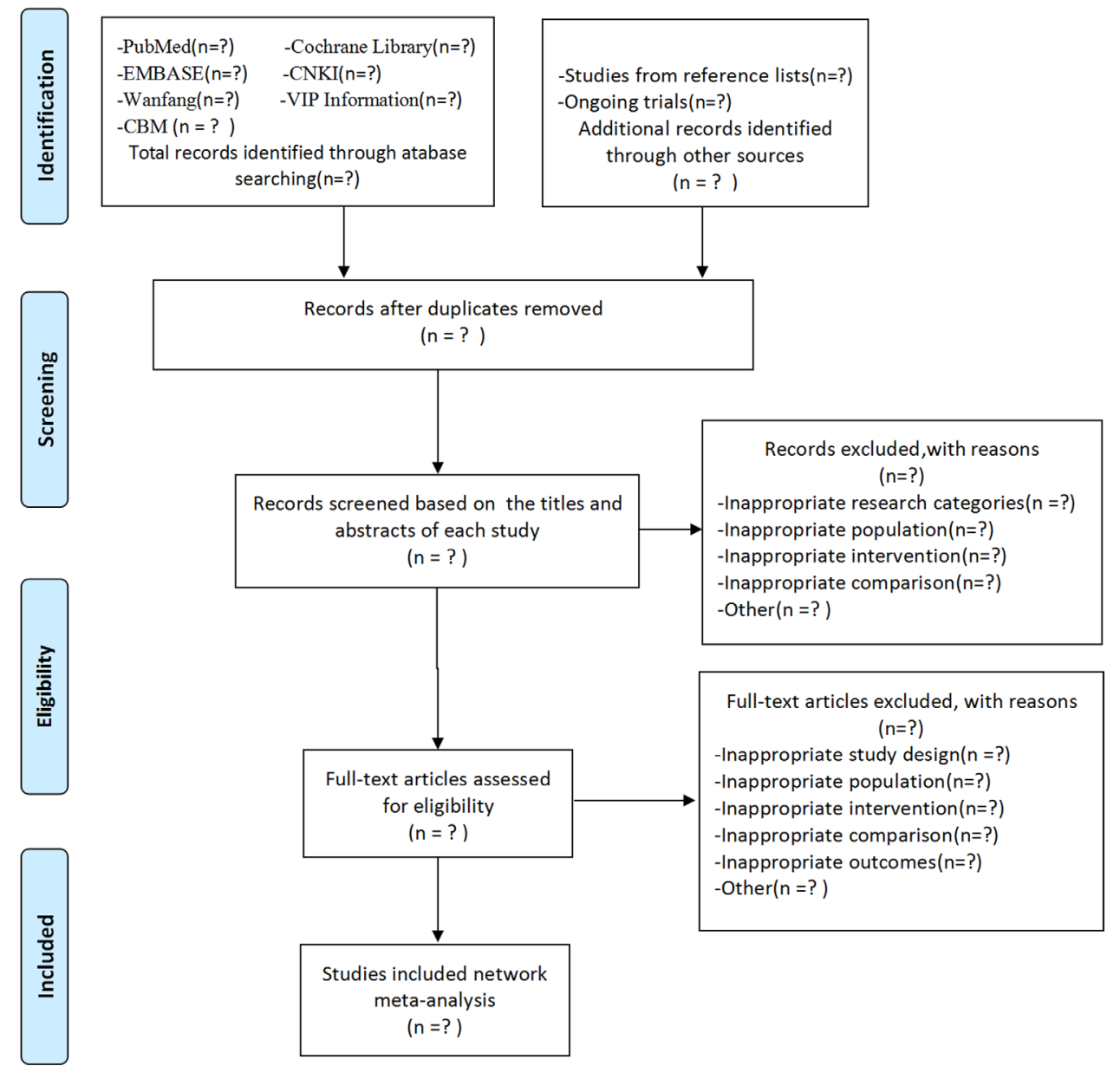

Figure 3 Proposed flowchart to depict the search process.

Two reviewers (Peng Xu and Kun Dong) will perform data extraction independently. Any disagreement will be resolved through discussion or consultation with a third author (Guan-lin Yang). We will contact original authors through email to request any missing information that may affect eligibility.

\section{Dealing with missing data}

We will try to get missing data by contacting research authors and discuss the reasons, extent, nature and how to deal with missing data in each study. For the dichotomous outcome, we will conduct sensitivity analyses using best-worst case scenario analysis to explore the potential impact of the missing data. For continuous outcomes, participants with missing outcome data will be excluded from the analysis.

\section{Assessment of risk of bias in the included studies}

The risk of bias will be performed by two raters independently (Zhen Hua and Feng-ting Zhai) based on the Cochrane risk of bias tool. ${ }^{35}$ It includes seven specific domains: (1) random sequence generation, (2) allocation concealment, (3) blinding of participants and personnel, (4) blinding of outcome data, (5) incomplete outcome data, (6) selective reporting and (7) other bias. We will manage to reach consensus between two raters on condition that there are any disagreement when assessing the risk of bias of the studies. If necessary, a third rater (Guan-lin Yang or Zhe Zhang) will resolve the disagreement.

\section{Data analysis}

Pairwise meta-analyses

For each direct treatment comparison, if no less than two RCTs, we will perform pairwise meta-analyses. We will use the OR to assess the effect size of dichotomous variables, while the effect size of continuous variables will be assessed using the mean difference (MD), if these continuous variables are reported using the same scale, standardised mean difference otherwise, both with $95 \% \mathrm{CrI}$ (see figure 1). Given that the methodological and clinical heterogeneity among the included studies will create statistical heterogeneity in turn, the random-effects (RE) model will be used to pool direct evidence for every direct treatment comparison. Because methodological and clinical diversity always exist in pairwise meta-analysis, obviously statistical heterogeneity is inevitable. ${ }^{36}$ And we will assess the heterogeneity both within and across those treatment comparisons by calculating the $\mathrm{I}^{2}$ and visual inspection of forest plots within every pairwise comparison. The interpretation of $\mathrm{I}^{2}$ will be based on 
the thresholds level as set forth in the Cochrane Collaboration. ${ }^{37}$ If there is important heterogeneity that influences result, we will perform meta-regression to examine potential effect moderators such as age of participants, sample size, duration of disease, course of treatment and study quality and so on. It is generally known that TCM research always involves the problem of syndrome differentiation and treatment. ${ }^{38}$ The oral Chinese patent medicines in the study were mainly for promoting blood circulation and removing blood stasis, benefiting Qi and nourishing Yin, regulating Qi and broadening chest to relieve pain. ${ }^{39}$ Besides, there are three types of UAP (rest angina pectoris, new-onset angina pectoris and accelerated angina pectoris) and the severity of each type is different. To avoid the bias, we will do subgroup analysis of syndrome types and types of UAP or perform meta-regression of syndrome types and types of UAP as potential effect moderators. Moreover, if there is considerable heterogeneity, especially when the direction of the effect is inconsistent, which indicates that the quantitative synthesis is inappropriate, we will do a general statistical description.

\section{Network meta-analyses}

Given that we expect between-study heterogeneity, we will perform a RE NMA. Considering that some of the therapeutic regimens available to treat UAP have not been compared in head-to-head RCT studies, we anticipate that some of the potential comparisons between the interventions will not have direct evidence. The common comparator will be routine treatment for UAP as it is the current criteria of treatment. If any trial forms nodes which are not connected to the network, we will exclude them. With the lack of direct evidence for the potential comparisons, we will perform adjusted indirect comparisons which will provide an estimate of the treatment effect. In the case of direct evidence, the NMA will be based on a mixed treatment comparison model generation and it will provide a combined estimate including direct comparison and indirect comparison evidence. ${ }^{40}$ We will perform the NMA using Markov Chain Monte Carlo method in a Bayesian hierarchical framework. And effect sizes will be reported using the OR or MD along with $95 \% \mathrm{Crl}$. A network diagram will be generated for each outcome. The network diagram is made up of lines and dots. In the diagram, the dots will represent every intervention, and the size of the dot will mean the amount of participants. The lines will indicate direct comparisons between different interventions and the thickness of the line will mean the amount of studies. ${ }^{41}$ For each outcome, we will present the contribution plots, which exposit the contribution of each direct comparison to the entire network as well as for each network estimate. ${ }^{42}$ The main characteristics of NMA are ranking analysis having the ability to rank the various treatments for each outcome. The surface under the cumulative ranking curve (SUCRA) values and the mean ranks will be used to rank the probabilities. ${ }^{43}$ The SUCRA values may range from $0 \%$ to $100 \%$, and the higher predicted value indicates that the treatment regimen is more effective.

\section{Assessment of inconsistency}

Inconsistency is differences between indirect and direct effect estimates for the same comparison, including loop inconsistency and design inconsistency. ${ }^{44}$ We will use the node-splitting method to evaluate the inconsistency between direct and indirect evidence locally. If $\mathrm{p}>0.05$, it suggests consistency between direct and indirect evidence. As we all know, tests for inconsistency have low power, ${ }^{45}$ and recent evidence has shown that only $2 \%-14 \%$ of evidence loops reported in the medical literature are expected to be inconsistent. ${ }^{46}$ Therefore, we must be cautious to interpret the statistical inference about inconsistency.

\section{Assessment of reporting bias}

A comparison-adjusted funnel plot and funnel plot for each treatment comparison and outcome (at least 10 trials ${ }^{48}$ will be constructed to assess the small-study effects and potential publication bias). ${ }^{49}$ And Egger's test will be used to assess the symmetry of the funnel plot. ${ }^{49}$ For the funnel plot, the horizontal axis will represent the difference between effect sizes and the comparison-specific summary effect. Due to the lack of small-study effects or publication bias, the comparison-adjusted funnel plot should be symmetric around the zero line.

\section{Model implementation}

In our NMA, we will fit our model using WinBUGS $14.3^{50}$ and Stata 14.0. The RE model with vague priors for multiarm trials will be used. ${ }^{43}$ We will use a Markov chain Monte Carlo method called Gibbs sampling to estimate the model parameters. Convergence will be found to be adequate after running 1000 samples. These samples are discarded as 'burn-in', and posterior summaries will be based on 100000 subsequent simulations. Production of network graphs, contribution plots and result figures and analyses for statistical evaluation of the inconsistency will be carried out in Stata 14.0 by the mvmeta and network command. ${ }^{44}$ We will also duplicate all analyses of the primary outcomes using the netmeta package in $\mathrm{R}$ software. ${ }^{51}$

\section{Quality of evidence}

We will assess the quality of evidence and the strength of recommendations for the main outcomes using the GRADE guidelines. ${ }^{52}$ There are five factors that can reduce the quality of evidence: study limitations (risk of bias), inconsistency, indirectness, publication bias and imprecision. Correspondingly, three factors can improve the quality of evidence: residual confounding, dose-response gradient and large magnitude of effect. The quality of evidence will be graded in four levels: very low, low, moderate, high. This step will be conducted using GRADEprofiler (GRADEpro3.6software). 


\section{Patient and public involvement}

No patients or public involvement will be involved in this study.

\section{DISCUSSION}

The proposed NMA will provide evidence of the comparative effectiveness and safety of oral Chinese patent medicines for UAP on the national essential drugs list of China. As we have seen, this NMA will be the first NMA in this respect. Because the findings of our study could be used to provide clinical recommendations for adults with UAP, it will interest a broad audience, including cardiologists, practice guideline developers, decision makers, researchers, policymakers and patients.

However, this NMA will have some limitations. Chinese medicine is widely used in China and also in East Asian countries, such as Korea and Japan. However, we will not search medical databases in languages such as Japanese and Korean, so excluding those studies may lead to language bias. In addition, the problem of clinical heterogeneity should not be ignored. If the scope of included studies is small, the ability to explore heterogeneity and conduct meta-regression could be limited. Moreover, we do not limit the dose and duration of the intervention, so we anticipate the dose and duration of the intervention in different clinical studies cannot be absolutely unified; however, the inconsistency will be reduced by setting subgroups or conducting meta-regression.

Nevertheless, this study is expected to provide a ranking of oral Chinese patent medicines for UAP. Based on this ranking of medicines, clinicians will make more accurate and optimal treatment decisions for adults with UAP. We will publish the findings and results of this study in a peer-reviewed journal.

\section{ETHICS AND DISSEMINATION}

Because of the nature of the study, no ethical approval and no informed consent are required. We will publish the findings and results of this study in a peer-reviewed journal.

\section{Author affiliations}

${ }^{1}$ Shandong Provincial Qianfoshan Hospital, The First Hospital Affiliated with Shandong First Medical University, Jinan, China

${ }^{2}$ The First Clinical Medical College, Shandong University of Traditional Chinese

Medicine, Jinan, China

${ }^{3}$ Evidence-based Medicine Center of Lanzhou University, Lanzhou, China

${ }^{4}$ Center of Rehabilitation Medicine, Sichuan University, Chengdu, China

${ }^{5}$ Biomedical Engineering, Hong Kong Polytechnic University, Kowloon, Hong Kong

${ }^{6}$ College of Traditional Chinese Medicine, Changchun University of Traditional

Chinese Medicine, Changchun, China

${ }^{7}$ Affiliated Hospital of Liaoning University of Traditional Chinese Medicine, Shenyang, China

${ }^{8}$ Liaoning Traditional Chinese Medicine of Jiahe, Shenyang, China

${ }^{9}$ Liaoning University of Traditional Chinese Medicine, Shenyang, China

${ }^{10}$ Key Laboratory of Ministry of Education for TCM Viscera-State Theory and

Applications, Liaoning University of Traditional Chinese Medicine, Liaoning, China
Contributors GY, ZZ and ZH conceptualised and designed the study. ZH and F-TZ drafted the manuscript protocol, and this was revised by ZZ, PX, CG and JT. The search strategy was developed by XD. The selection criteria, data abstraction criteria and risk of bias assessment strategy were developed by all of the authors. $\mathrm{CG}, \mathrm{FZ}$ and $\mathrm{S}$-jL will perform the search strategy, and PX and KD will screen the potential studies, and $\mathrm{ZH}$ and F-TZ will assess the risk of bias. JT provided statistical expertise. All the reviewers have read and approved the final manuscript.

Funding This study was supported by grants from the Clinical capacity-building project of Liaoning Province in China (LNCCC-B05-2014) and by the Open fund of Key Laboratory of Ministry of Education for TCM Viscera-State Theory and Applications, Liaoning University of Traditional Chinese Medicine.

Competing interests None declared.

Patient consent for publication Not required.

Provenance and peer review Not commissioned; externally peer reviewed.

Open access This is an open access article distributed in accordance with the Creative Commons Attribution Non Commercial (CC BY-NC 4.0) license, which permits others to distribute, remix, adapt, build upon this work non-commercially, and license their derivative works on different terms, provided the original work is properly cited, appropriate credit is given, any changes made indicated, and the use is non-commercial. See: http://creativecommons.org/licenses/by-nc/4.0/.

\section{REFERENCES}

1. Braunwald E, Jones RH, Mark DB, et al. Diagnosing and managing unstable angina. agency for health care policy and research. Circulation 1994;90:613-22.

2. Benjamin EJ, Virani SS, Callaway CW, et al. Heart disease and stroke Statistics-2018 update: a report from the American heart association. Circulation 2018;137:e67-492.

3. Benjamin EJ, Blaha MJ, Chiuve SE, et al. Heart disease and stroke Statistics-2017 update: a report from the American heart association. Circulation 2017;135:e146-603.

4. Mozaffarian D, Benjamin EJ, Go AS, et al. Heart disease and stroke Statistics-2016 update: a report from the American heart association. Circulation 2016;133:e38-60.

5. Libby P. Mechanisms of acute coronary syndromes and their implications for therapy. N Engl J Med 2013;368:2004-13.

6. Badimon L, Padró T, Vilahur G. Atherosclerosis, platelets and thrombosis in acute ischaemic heart disease. Eur Heart $J$ 2012;1:60-74.

7. Davi G, Patrono C. Platelet activation and atherothrombosis. N Engl $J$ Med 2007;357:2482-94.

8. Amsterdam EA, Wenger NK, Brindis RG, et al. 2014 AHA/ACC guideline for the management of patients with non-ST-elevation acute coronary syndromes: a report of the American College of Cardiology/American heart association Task force on practice guidelines. Circulation 2014;130:e344-426.

9. Roffi M, Patrono C, Collet J-P, et al. 2015 ESC guidelines for the management of acute coronary syndromes in patients presenting without persistent ST-segment elevation: Task force for the management of acute coronary syndromes in patients presenting without persistent ST-segment elevation of the European Society of cardiology (ESC). Eur Heart $J$ 2016;37:267-315.

10. Chinese Society of cardiology, editorial board of Chinese Journal of cardiovascular. guidelines for diagnosis and treatment of non-STsegment elevation acute coronary syndromes (2016). Chin J Cardiol 2017;5:359-76.

11. Breuckmann F, Hochadel M, Darius $\mathrm{H}$, et al. Guideline-adherence and perspectives in the acute management of unstable angina Initial results from the German chest pain unit registry. J Cardiol 2015;66:108-13.

12. Goss F, Brachmann J, Hamm CW, et al. High adherence to therapy and low cardiac mortality and morbidity in patients after acute coronary syndrome systematically managed by office-based cardiologists in Germany: 1-year outcomes of the ProAcor study. Vasc Health Risk Manag 2017;13:127-37.

13. Taggart $D$. Tailor treatment to the patient in stable angina. Practitioner 2011;255:25-8.

14. Chen $\mathrm{KJ}, \mathrm{Xu} \mathrm{H}$. The integration of traditional Chinese medicine and Western medicine. European Rev 2003;11:225-35.

15. Xutian S, Cao D, Wozniak J, et al. Comprehension of the unique characteristics of traditional Chinese medicine. Am J Chin Med 2012;40:231-44.

16. Hao P, Jiang F, Cheng J, et al. Traditional Chinese medicine for cardiovascular disease: evidence and potential mechanisms. J Am Coll Cardiol 2017;69:2952-66. 
17. Tindle HA, Davis RB, Phillips RS, et al. Trends in use of complementary and alternative medicine by US adults: 1997-2002. Altern Ther Health Med 2005;11:42-9.

18. Claxton M. Culture, health and civilization. In: Leander B, ed. Culture and health. Paris: UNESCO Pub, 1996: 17-46.

19. National Health Commission of the People's Republic of China. The National essential drugs list of China (2018 edition). Beijing: People's Medical Publishing House, 2018.

20. Li S. The Use of Suxiaojiuxinwan in Southeast Asia (Golden Triangle Area) - A Glimpse. Chinese Traditional Patent Medicine 1995;17.

21. Sung HJ, Shin HK. Comparision and research on policy of traditional medicine in Korea and three countries of East Asia. Korean: Korean Institute of Oriental Medicine, 1997.

22. World Health Organization. WTO traditional medicine strategy 20022005. World Health Organization, 2002.

23. Yang X, Xiong X, Yang G, et al. Chinese patent medicine Xuefu Zhuyu capsule for the treatment of unstable angina pectoris: a systematic review of randomized controlled trials. Complement Ther Med 2014;22:391-9.

24. Wu T, Harrison RA, Chen XY, et al. Tongxinluo (Tong xin luo or Tongxin-luo) capsule for unstable angina pectoris. Cochrane Database Syst Rev 2006;13.

25. Yang $X$, Xiong $X$, Wang $H$, et al. Xuesaitong soft capsule (Chinese patent medicine) for the treatment of unstable angina pectoris: a meta-analysis and systematic review. Evid Based Complement Alternat Med 2013;2013:1-7.

26. Zoccai GB. Network meta-analysis: evidence synthesis with mixed treatment comparison. New York: Nova Science Publishers Inc, 2014.

27. Shamseer L, Moher D, Clarke M, et al. Preferred reporting items for systematic review and meta-analysis protocols (PRISMA-P) 2015: elaboration and explanation. BMJ 2015;349:g7647.

28. Hutton B, Salanti G, Caldwell DM, et al. The PRISMA extension statement for reporting of systematic reviews incorporating network meta-analyses of health care interventions: checklist and explanations. Ann Intern Med 2015;162:777-84.

29. The Joint International Society and Federation of Cardiology/ World Health Organization task force on standardization of clinical nomenclature. Nomenclature and criteria for diagnosis of ischemic heart disease. Circulation 1979;59:607-9.

30. Chinese Society of Cardiovascular Diseases of Chinese Medical Association. Diagnosis and treatment of unstable angina pectoris. Chin J Cardiol 2000;06:8-11.

31. Chinese Society of Cardiovascular Diseases of Chinese Medical Association. Editorial Committee of Chinese Journal of Cardiology. Unstable angina and non-ST segment elevation myocardial infarction diagnosis and treatment guidelines. Chin J Cardiol 2007;35:295-304.

32. Ge J, Xu Y. Internal medicine. 8th edn. Beijing: People's Medical Publishing House, 2014.

33. Symposium on treatment of angina pectoris and arrhythmia of coronary heart disease with integrated traditional Chinese and Western medicine. Coronary heart disease, angina pectoris and electrocardiogram evaluation criteria (1979, Shanghai). Chinese Pharmaceutical Affairs 1987;02:17-20.
34. Corp A. Introdution of NoteExpress, 2018. Available: http://www. inoteexpress.com/index.htm

35. Higgins JPT, Green S. Cochrane Handbook for systematic reviews of interventions version 5.1.0 (updated March 2011). The Cochrane collaboration, 2011. Available: http://www.handbook.cochrane.org/

36. Higgins JPT, Thompson SG, Deeks JJ, et al. Measuring inconsistency in meta-analyses. BMJ 2003;327:557-60.

37. Higgins JPT, Altman DG. Chapter 9: Analysing data and undertaking meta-analyses. In: Deeks JJ, Higgins JPT, eds. Cochrane Handbook for systematic reviews of interventions. Chichester, UK: John Wiley \& Sons, 2011.

38. Jiang M, Lu C, Zhang C, et al. Syndrome differentiation in modern research of traditional Chinese medicine. J Ethnopharmacol 2012;140:634-42.

39. Tian P, Li J. Treatment methods of traditional Chinese medicine for unstable angina of coronary heart disease. Jilin Journal of Chinese Medicine 2019;39:972-6.

40. Mills EJ, Thorlund K, loannidis JPA. Demystifying trial networks and network meta-analysis. BMJ 2013;346:f2914.

41. Catalá-López F, Tobías A, Cameron C, et al. Network meta-analysis for comparing treatment effects of multiple interventions: an introduction. Rheumatol Int 2014;34:1489-96.

42. Chaimani A, Higgins JPT, Mavridis D, et al. Graphical tools for network meta-analysis in STATA. PLoS One 2013;8:e76654.

43. Salanti G, Ades AE, loannidis JPA. Graphical methods and numerical summaries for presenting results from multiple-treatment metaanalysis: an overview and tutorial. J Clin Epidemiol 2011;64:163-71.

44. White IR, Barrett JK, Jackson D, et al. Consistency and inconsistency in network meta-analysis: model estimation using multivariate metaregression. Res Synth Methods 2012;3:111-25.

45. Veroniki AA, Mavridis D, Higgins JPT, et al. Characteristics of a loop of evidence that affect detection and estimation of inconsistency: a simulation study. BMC Med Res Methodol 2014;14:106.

46. Veroniki AA, Vasiliadis HS, Higgins JPT, et al. Evaluation of inconsistency in networks of interventions. Int $\mathrm{J}$ Epidemiol 2013;42:332-45.

47. Song F, Xiong T, Parekh-Bhurke S, et al. Inconsistency between direct and indirect comparisons of competing interventions: metaepidemiological study. BMJ 2011;343:d4909.

48. Higgins JPT, Altman DG, Higgins JPT, et al. Chapter 8: Assessing risk of bias in included studies. In: Cochrane Handbook for systematic reviews of interventions. Chichester, UK: John Wiley \& Sons, 2011.

49. Egger M, Davey Smith G, Schneider M, et al. Bias in meta-analysis detected by a simple, graphical test. BMJ 1997;315:629-34.

50. Lunn DJ, Thomas A, Best N, et al. WinBUGS-A Bayesian modeling frame work: concepts, structure, and extensibility. Stat Comput 2000;10:325-37.

51. Schwarzer G. Network meta-analysis. In: Schwarzer G, Carpenter JR, Rücker G, eds. Meta-analysis with R. Berlin. Heidelberg: Springer, 2015: 187-216.

52. Guyatt G, Oxman AD, Akl EA, et al. GRADE guidelines: 1. Introduction-GRADE evidence profiles and summary of findings tables. J Clin Epidemiol 2011;64:383-94. 